\title{
Erratum to: Berardinelli Seip congenital lipodystrophy presenting with neonatal diabetes mellitus due to a mutation in the AGPAT2 gene
}

\author{
V. Poovazhagi $\cdot$ S. Shanthi $\cdot$ S. Jahnavi $\cdot$ V. Radha $\cdot$ V. Mohan
}

Published online: 19 June 2013

(C) Research Society for Study of Diabetes in India 2013

Erratum to: Int J Diabetes Dev Ctries

\section{DOI 10.1007/s13410-012-0099-6}

In the original publication of this article, published in Vol 33 No 1 pp 66-68, the affiliation of V. Poovazhagi and S. Shanthi was wrong. It should have been "Institute of Child Health and Hospital for Children, Chennai Tamil Nadu, India" instead of "Madras Diabetes Research Foundation, Chennai, Tamil Nadu, India". 10.1007/s13410-012-0099-6.

V. Poovazhagi $(\bowtie) \cdot$ S. Shanthi Institute of Child Health and Hospital for Children, Chennai,

Tamil Nadu, India

e-mail: poomuthu@yahoo.com

S. Jahnavi $\cdot$ V. Radha $\cdot$ V. Mohan

Madras Diabetes Research Foundation, Chennai,

Tamil Nadu, India 